\title{
Structure and Optical Properties of Silicon Nanocrystals Embedded in Amorphous Silicon Thin Films Obtained by PECVD
}

\author{
B. M. Monroy, ${ }^{1}$ A. Remolina, ${ }^{1}$ M. F. García-Sánchez, ${ }^{1}$ A. Ponce, ${ }^{2}$ \\ M. Picquart, ${ }^{3}$ and G. Santana ${ }^{1}$ \\ ${ }^{1}$ Instituto de Investigaciones en Materiales, Universidad Nacional Autónoma de México, \\ A.P. 70-360, 04510 Coyoacán, DF, Mexico \\ ${ }^{2}$ Centro de Investigación en Química Aplicada, Boulevard Enrique Reyna Hermosillo no.140, Saltillo, 25290, Coahuila, Mexico \\ ${ }^{3}$ Departamento de Física, Universidad Autónoma Metropolitana Iztapalapa, A.P. 55-534, 09340 Iztapalapa, DF, Mexico
}

Correspondence should be addressed to B. M. Monroy, marel@iim.unam.mx

Received 28 May 2010; Accepted 10 November 2010

Academic Editor: Jaetae Seo

Copyright (๑) 2011 B. M. Monroy et al. This is an open access article distributed under the Creative Commons Attribution License, which permits unrestricted use, distribution, and reproduction in any medium, provided the original work is properly cited.

\begin{abstract}
Silicon nanocrystals embedded in amorphous silicon matrix were obtained by plasma enhanced chemical vapor deposition using dichlorosilane as silicon precursor. The RF power and dichlorosilane to hydrogen flow rate ratio were varied to obtain different crystalline fractions and average sizes of silicon nanocrystals. High-resolution transmission electron microscopy images and RAMAN measurements confirmed the existence of nanocrystals embedded in the amorphous matrix with average sizes between 2 and $6 \mathrm{~nm}$. Different crystalline fractions (from 12\% to 54\%) can be achieved in these films by regulating the selected growth parameters. The global optical constants of the films were obtained by UV-visible transmittance measurements. Effective band gap variations from 1.78 to $2.3 \mathrm{eV}$ were confirmed by Tauc plot method. Absorption coefficients higher than standard amorphous silicon were obtained in these thin films for specific growth parameters. The relationship between the optical properties is discussed in terms of the different internal nanostructures of the samples.
\end{abstract}

\section{Introduction}

For several decades, hydrogenated amorphous silicon (a$\mathrm{Si}: \mathrm{H})$ has been investigated as a possible candidate for the production of inexpensive solar cells $[1,2]$. However, the expected efficiency and stability of a-Si:H solar cells has not yet been achieved. The technological utility of this material is hampered by the creation of metastable electronic defects when excess free carriers are generated by light absorption (Staebler-Wronski effect) [3]. This light-induced degradation of a-Si:H depends mainly on changes in the hydrogen bonding configuration and the presence of defects in the films.

In the last decade, Roca i Cabarrocas et al. introduced polymorphous silicon (pm-Si) using silane $\left(\mathrm{SiH}_{4}\right)$ as silicon precursor and they obtained a new material characterized by the presence of dispersed nanocrystallines embedded in an amorphous network that is more relaxed than that found in standard a-Si:H [4]. It has already been shown that this material has better transport properties (enhanced hole mobility and a smaller density of metastable electronic defects) and improved photo stability with respect to a-Si:H $[5,6]$, thus making it a likely candidate for high efficiency solar cells. There has been considerable research on the structural characteristics and optoelectronic properties of this material [5-9].

The expectation is that replacing the a-Si:H by different forms of pm-Si thin films could reduce the cost of "solar electric power" by increasing the efficiency and stability of silicon-based thin film photovoltaic devices [7]. However, there are some critical issues that remain to be addressed to achieve the best performance of pm-Si in a photovoltaic device: (i) control of the nanocrystallite size distribution and density in the amorphous matrix which determines the optoelectronic properties of $\mathrm{pm}-\mathrm{Si}$, (ii) control of the hydrogen incorporation in the films and Medium Range Order (MRO) of the amorphous matrix to minimize the Staebler-Wronski effect, and (iii) optimization of the processing of pm-Si thin 


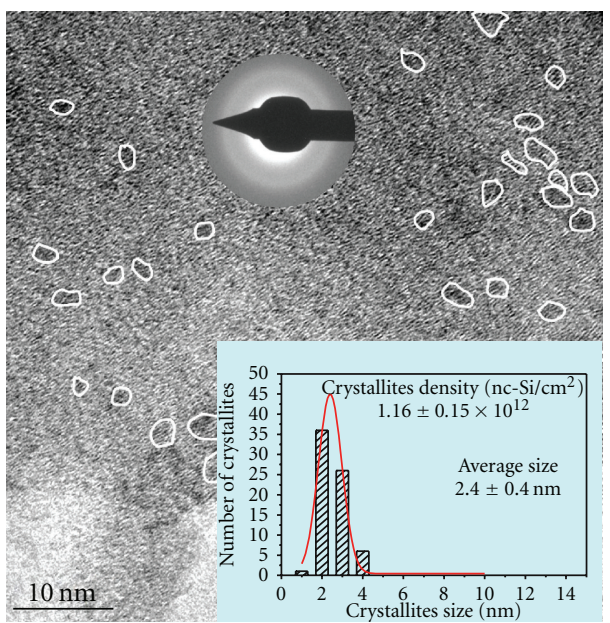

(a) sample with $R=0.1$ and 10 Watt of RF power

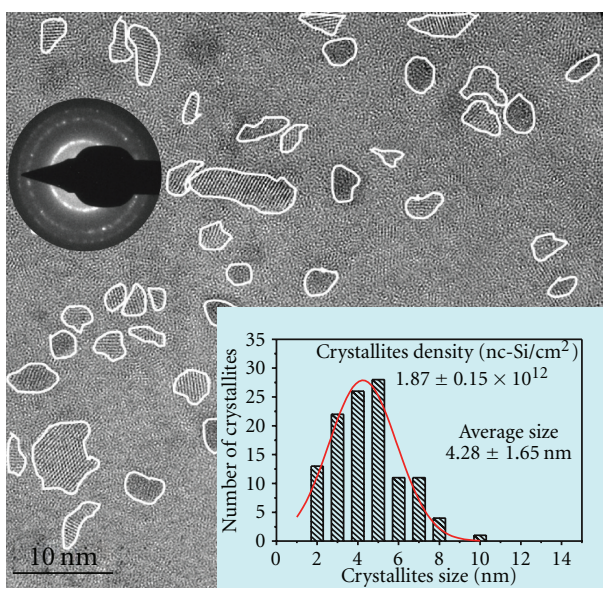

(c) sample with $R=0.1$ and 150 Watt of RF power

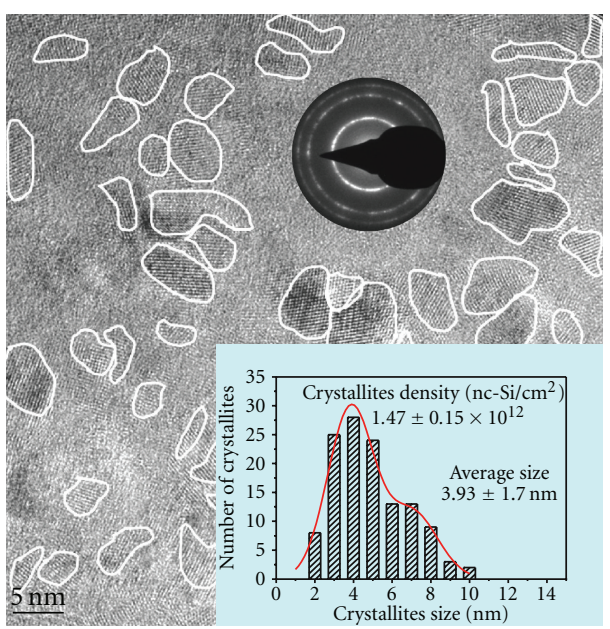

(e) sample with $R=0.05$ and 50 Watt of RF power

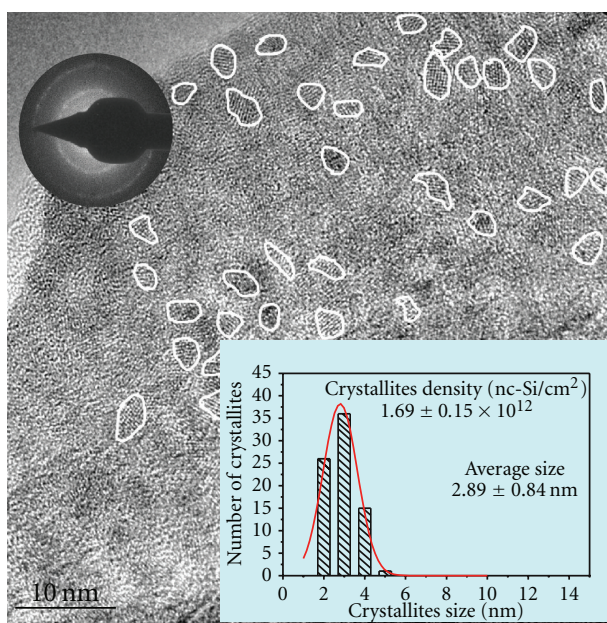

(b) sample with $R=0.1$ and 50 Watt of RF power

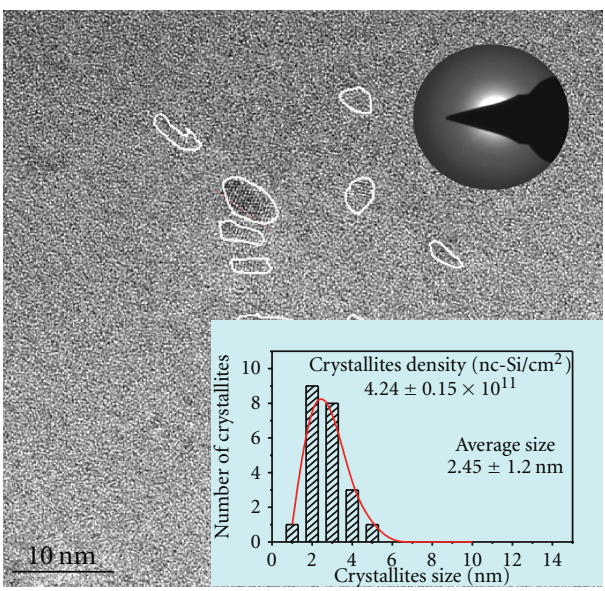

(d) sample with $R=0.05$ and 10 Watt of RF power

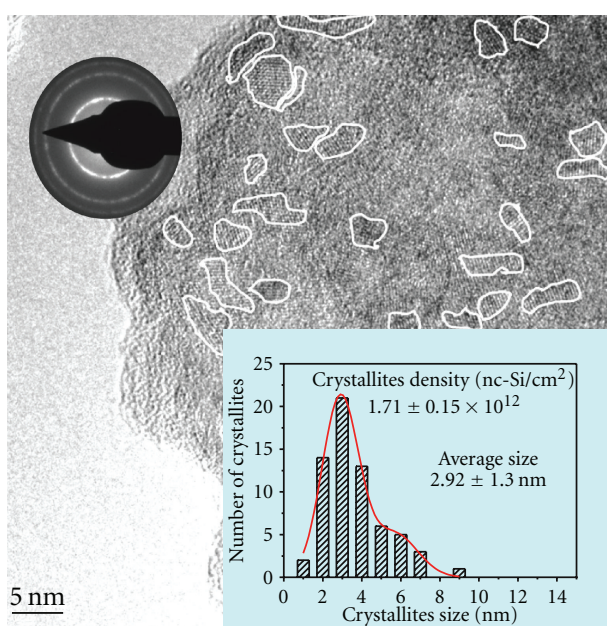

(f) sample with $R=0.05$ and 150 Watt of RF power

FIGURE 1: Representative HRTEM images for six samples grown with different dichlorosilane to hydrogen flow rate ratios $(R)$ and RF powers. The circular inserts show the Small Area Diffraction Patterns of the samples, and the square inserts show the size distributions and number densities of the silicon nanocrystalline inclusions. 


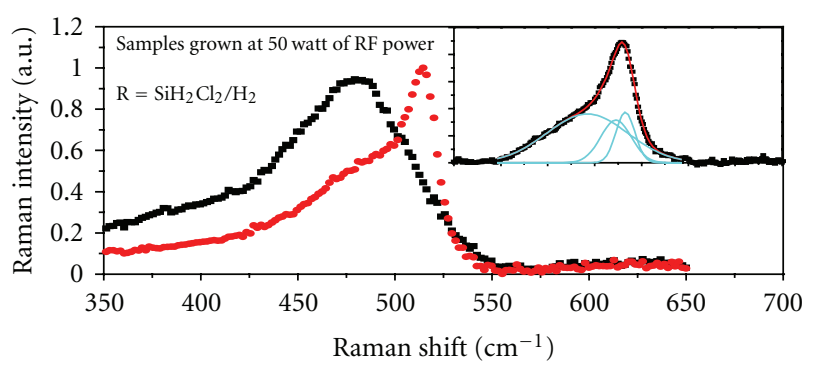

- $R=0.1$

- $R=0.05$

(a) Samples Grown at 50 Watt of RF Power

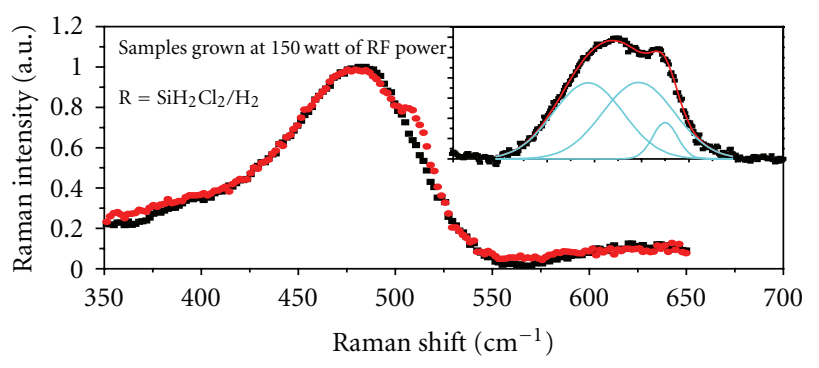

- $R=0.1$

- $R=0.05$

(b) Samples Grown at 150 Watt of RF Power

FIGURE 2: Comparison of Raman spectra of polymorphous silicon thin films grown with different $R=\operatorname{Fr}\left[\mathrm{SiH}_{2} \mathrm{Cl}_{2}\right] / \mathrm{Fr}\left[\mathrm{H}_{2}\right]$ and RF powers of (a) 50 Watts and (b) 150 Watts. The insert shows an example of the Raman spectra deconvolution in three peaks.

films to comply with the requirements of the photovoltaic industry.

Previously, we have reported the growth of different silicon alloys by plasma enhanced chemical vapor deposition (PECVD) using dichlorosilane $\left(\mathrm{SiH}_{2} \mathrm{Cl}_{2}\right)$ as silicon precursor [10-12]. Due to the chlorine chemistry introduced in the deposition process, it is possible to obtain nanocrystalline silicon inclusions at low deposition temperatures and regulate the hydrogen content of the films, leading to an increased chemical stability of the material. Since the optoelectronic properties of this particular type of silicon thin films, which we also name polymorphous silicon (pm-Si), depend on the size and density of the silicon nanocrystallites (nc$\mathrm{Si}$ ) embedded in the amorphous matrix, a study for the determination of the average crystallite sizes and density as a function of growth parameter becomes essential.

The aim of this paper is to show the variations of the optoelectronics properties in $\mathrm{pm}-\mathrm{Si}$ as a function of the nanocrystalline fraction, which can be regulated by means of specific growth parameters, as the RF power of the PECVD process and through the dichlorosilane to hydrogen flow rate ratio $\left(R=\operatorname{Fr}\left[\mathrm{SiH}_{2} \mathrm{Cl}_{2}\right] / \mathrm{Fr}\left[\mathrm{H}_{2}\right]\right)$. Depending on their absorption properties and effective band gap, these materials can be suitable to apply in different parts of amorphous silicon thin film solar cell devices.

\section{Experimental Procedure}

The samples were prepared using a conventional PECVD system with parallel plates of $150 \mathrm{~cm}^{2}$ in area and $1.5 \mathrm{~cm}$ apart, activated by a $13.56 \mathrm{MHz}$ RF signal. The system is described in more detail elsewhere [13]. The thin films were deposited simultaneously on high resistivity (100) singlecrystalline silicon, fuse silica (quartz), and $\mathrm{NaCl}$ substrates to facilitate the different characterizations. Prior to deposit, the substrates were subjected to a standard cleaning procedure, immediately before loading into the deposition chamber. Ultrahigh purity $\mathrm{SiH}_{2} \mathrm{Cl}_{2}$ and $\mathrm{H}_{2}$ were used as precursor gases. In all cases, the substrate temperature was kept constant at $200^{\circ} \mathrm{C}$ and an argon flow rate of $50 \mathrm{sccm}$ was used as diluent gas. The deposition time and pressure were fixed at $30 \mathrm{~min}$ and $500 \mathrm{mTorr}$, respectively. Plasma powers of $10 \mathrm{~W}, 25 \mathrm{~W}, 50 \mathrm{~W}, 100 \mathrm{~W}$ and $150 \mathrm{~W}$ were tested. Two different dichlorosilane to hydrogen flow rate ratios $(\mathrm{R}=$ $\left.\operatorname{Fr}\left[\mathrm{SiH}_{2} \mathrm{Cl}_{2}\right] / \mathrm{Fr}\left[\mathrm{H}_{2}\right]\right)$ were used, 0.05 and 0.1 , while the hydrogen flow was fixed at $50 \mathrm{sccm}$ in all cases. The thickness of the films over all substrates was determined by a Sloan Dektak IIA surface profile measuring system. The deposition conditions, film thickness, and growth rate of the studied samples are summarized in Table 1. The effect of varying the hydrogen dilution and RF power was to change the plasma chemistry and investigate its effect on the optoelectronic properties of the pm-Si films.

High-resolution transmission electron microscopy (HRTEM) studies have been carried out in a TITAN-300 kV field emission gun microscope, which has a symmetrical condenser-objective lens type S-TWIN $(\mathrm{Cs}=1.3 \mathrm{~mm})$. The HRTEM images have been registered in a CCD camera near the Scherzer focus and subsequently analyzed using Digital Micrograph software package. It is important to notice that the samples deposited over $\mathrm{NaCl}$ substrates were used for HRTEM analysis. In each case, the substrate was dissolved in distilled water and the film was collected on a Cu grid. In this way, we ensured that no modification of the film structure is produced, as could be the case in conventional ion milling techniques used to prepare samples for HRTEM. For Raman and UV-visible measurements, the samples deposited over quartz were used. The Raman spectra were recorded using a T64000 Jobin-Yvon Horiba triple monochromator using the $\times 100$ microscope objective. The excitation source was the $514.5 \mathrm{~nm}$ line from an $\mathrm{Ar}^{+}$Lexel laser. All the measurements were performed at room temperature in open air. The sample was irradiated at a power of $20 \mathrm{~mW}$ at the laser head. Ten accumulations with an integration time of $1 \mathrm{~min}$ were performed on each sample in the range $10-1200 \mathrm{~cm}^{-1}$. The Raman signal was detected by a cooled CCD. Transmittance measurements were recorded in a UV-Vis spectrometer Jasco $\mathrm{V}-630$ in a two-beam configuration.

\section{Results and Discussion}

3.1. HRTEM. All samples were studied by HRTEM with the aim of determining the sizes, shape, and density of crystallites embedded in the amorphous matrix. Six representative HRTEM images of samples with different nanostructures 


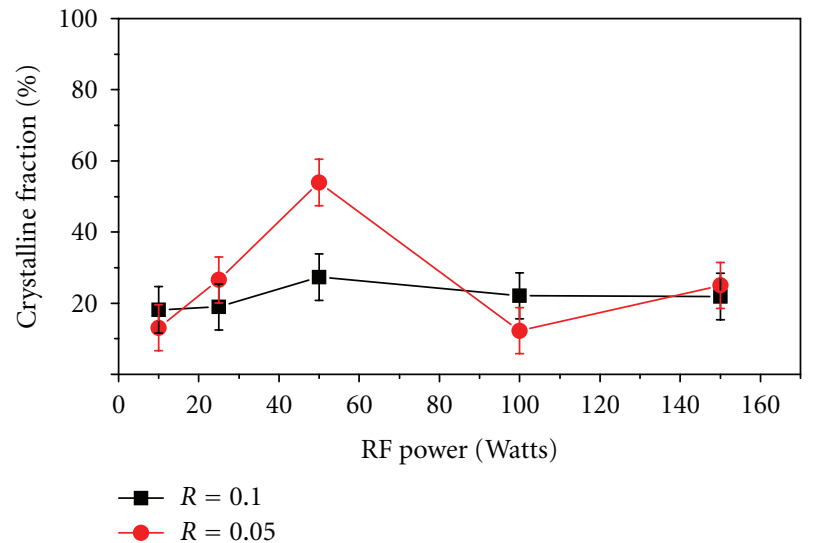

(a)

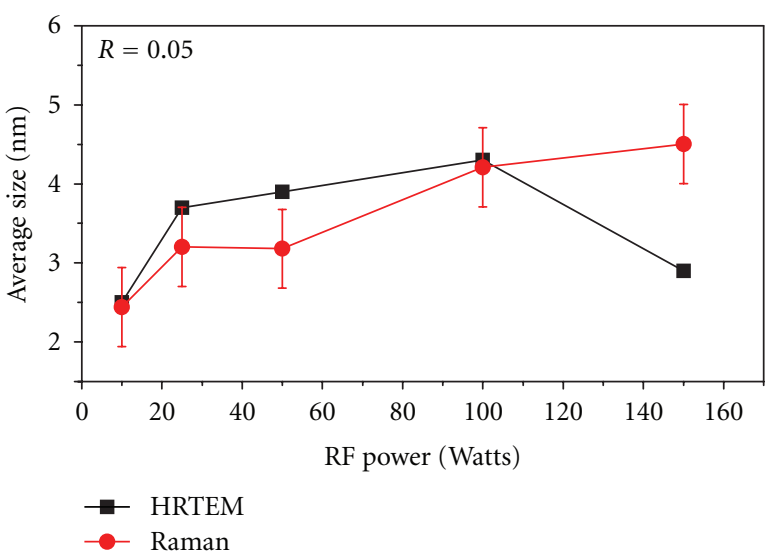

(b)

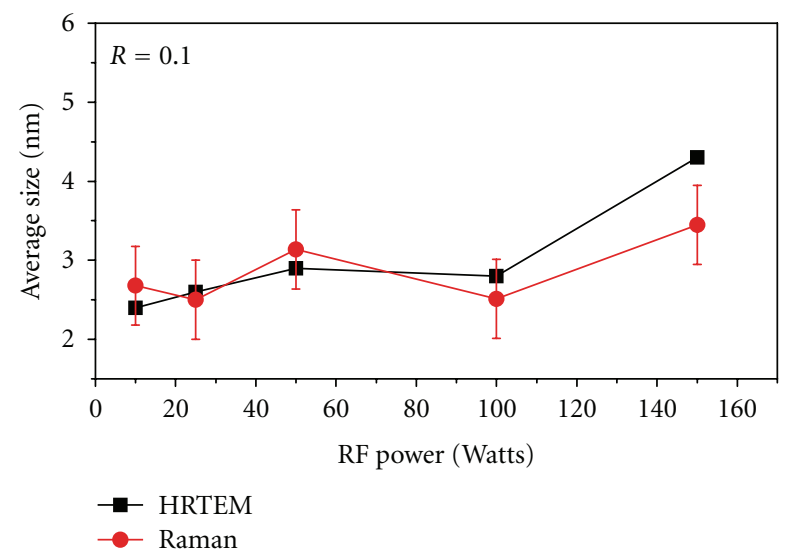

(c)

FIGURE 3: (a) Crystalline fraction and average sizes of silicon nanocrystallites obtained by Raman and HRTEM for samples grown with dichlorosilane to hydrogen flow rate ratios of (b) $R=0.05$ and (c) $R=0.1$, as a function of the RF power.

deposited with 10, 50, and 150 Watts of RF power, and flow rates $R$ of 0.05 and 0.1 are shown in Figure 1. As can be observed in the figure, the investigated samples contain randomly oriented crystallites of different sizes and shapes. Such nanometric ordered domains are indicated by a surrounding white line in the figure. Selected Area Electron Diffraction (SAED) patterns from the regions analyzed by HRTEM are included as circular inserts in Figure 1. As the dimensions and density of crystallites increase (Figures 1(c), $1(\mathrm{e})$ and $1(\mathrm{f})$ ), sharp diffraction rings become more evident. These patterns are representative of nanocrystalline materials $[7,14]$. On the other hand, when the size and density of crystallites is small, only diffuse rings characteristic of the amorphous matrix can be distinguished (Figures 1(a), 1(b), and $1(\mathrm{~d})$ ). For these samples, the ordered domains were limited to only 5 to 10 planes, showing that the crystallite dimension in one of its axis is between 2 to $6 \mathrm{~nm}$. These small nc-Si tend to be predominantly spherical as shown in Figure 1(a), while the larger crystallites (Figures 1(c), 1(e) and $1(f)$ ) exhibited various shapes and their dimensions can be in the order of $10 \mathrm{~nm}$. The density of nanocrystallites increases slightly with the increase of both $R$ and the
RF power. A more drastic effect is observed in the size distribution as shown in the inserts of Figure 1. For high RF powers, the size distribution is considerably broadened and it has no longer a Gaussian shape.

The different nanostructures observed by HRTEM can be explained in terms of the chlorine chemistry introduced in the deposition process. As was described in detail in previous works [10-12], metastable $\mathrm{SiCl}_{x} \mathrm{H}_{y}$ precursors that incorporate to the surface are highly reactive with impinging atomic hydrogen $\left(\mathrm{H}_{\mathrm{at}}\right)$. The Si-Si bond breaking by atomic hydrogen enhances the chemical reactivity of SiCld (d: dangling bond) and/or SiHCl-related complexes that promotes the creation of nucleation sites for nc-Si [15].

The relative amount of chlorine related species and atomic hydrogen depends importantly on the dichlorosilane to hydrogen flow rate ratio $(R)$ and the RF power. We can explain the irregular shapes of the samples with bigger nanocrystallites (Figures 1(c), 1(e), and 1(f)) in terms of the local heating induced by $\mathrm{HCl}$ formation. From the HRTEM images, it seems as if they were crystallized areas and not crystallites embedded in amorphous matrix. This is an important difference from previous reports where the silicon 


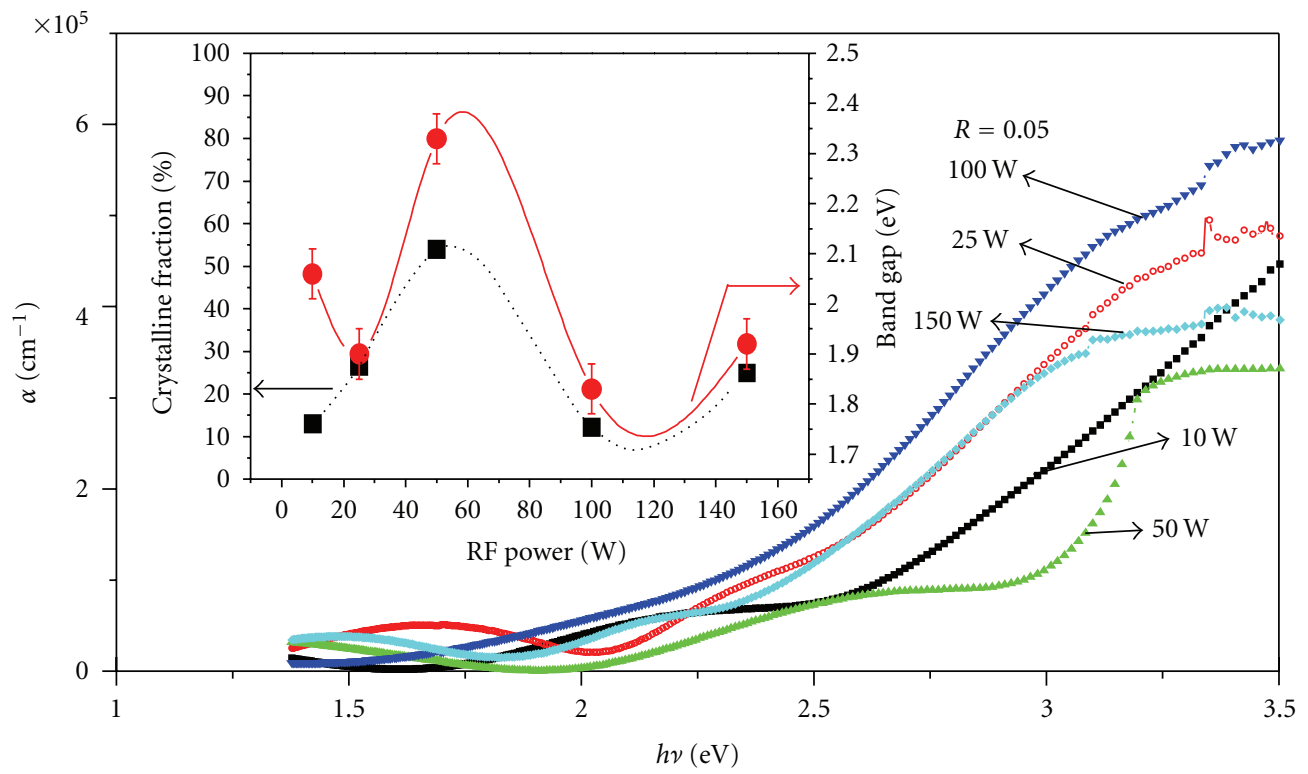

(a)

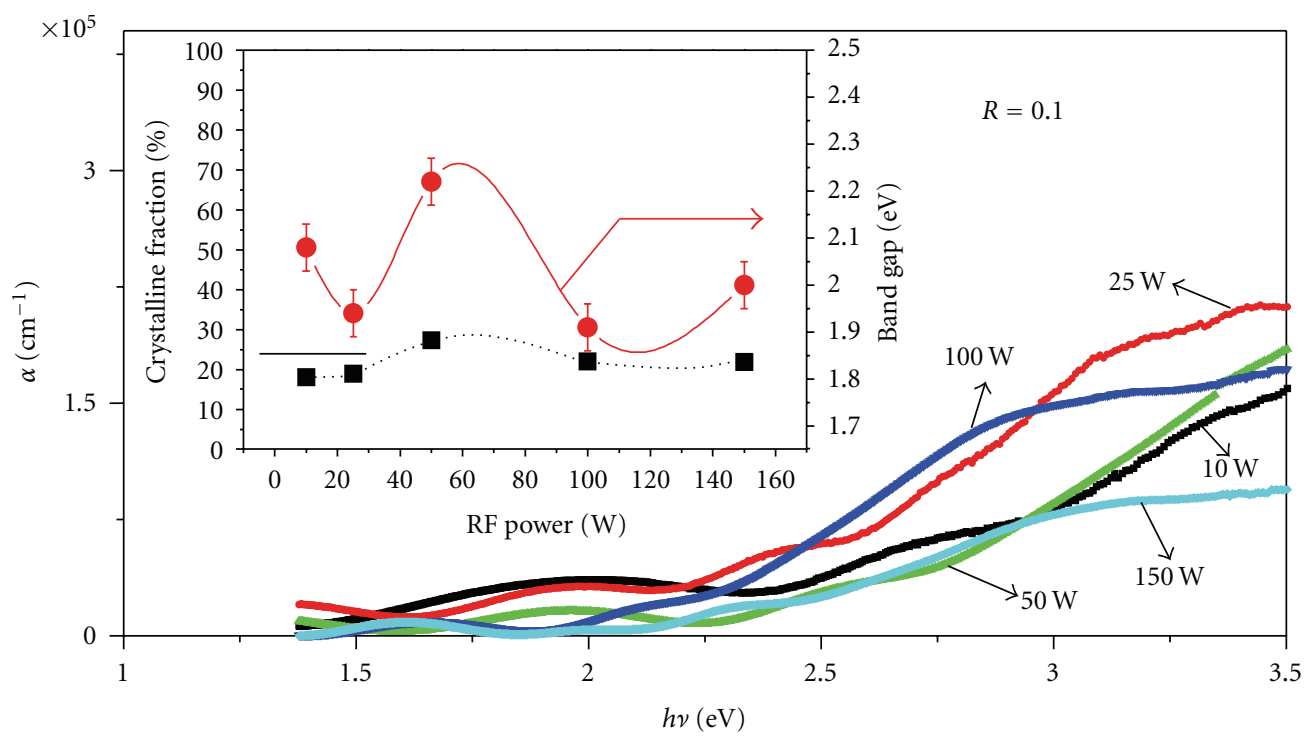

(b)

FIGURE 4: Absorption coefficient spectra for samples grown with different $R=\operatorname{Fr}\left[\mathrm{SiH}_{2} \mathrm{Cl}_{2}\right] / \mathrm{Fr}\left[\mathrm{H}_{2}\right]$ and RF power. The inserts show de Tauc optical band gap and crystalline fraction for the different samples as a function of the RF power.

nanocrystals in pm-Si thin films are primarily formed in the plasma region, and they incorporate to the a-Si matrix [4-6, 16]. The increment of nc-Si nucleation sites, $\mathrm{HCl}$ extraction reactions, and local heating with RF power is expected to cause a broader nc-Si size distribution during the deposition process, as is observed in Figure 1.

3.2. Raman Spectroscopy. We performed Raman Spectroscopy (RS) in order to describe quantitatively the variations of the crystalline volume fraction $\left(X_{C}\right)$ of the films. It is important to note that the Raman collection depth is less than $100 \mathrm{~nm}$ (because the excitation was done at
$514 \mathrm{~nm})$. Since all the samples are thicker than this limit, the analyzed volume (or integrated cross-section absorption) is similar in all cases. We also obtained the average crystallite size to compare with the HRTEM results. In general, it is assumed that for amorphous/crystalline mixed phase silicon thin films, such as pm-Si, the Raman spectrum consists of two contributions to the transverse optical (TO) mode peak: a sharp peak around $520 \mathrm{~cm}^{-1}$ and a broad peak around $480 \mathrm{~cm}^{-1}$ which correspond to the microcrystalline and amorphous phases, respectively. In addition, considering the strong phonon confinement due to the nanosize of the crystals, the phonon peak of c-Si is shifted from its 
TABLE 1: Films thickness and growth rate of polymorphous silicon thin films deposited by PECVD with different $R=\mathrm{Fr}[\mathrm{SiH} 2 \mathrm{Cl} 2] / \mathrm{Fr}[\mathrm{H} 2$ ] and RF powers.

\begin{tabular}{lcccc}
\hline Plasma power $(\mathrm{W})$ & $\begin{array}{c}R=0.05 \\
\text { Films thickness }(\mathrm{nm}) \pm 5 \mathrm{~nm}\end{array}$ & $\begin{array}{c}R=0.1 \\
\text { Growth rate }(\AA / \mathrm{s})\end{array}$ & $\begin{array}{c}\text { Films thickness }(\mathrm{nm}) \pm 5 \mathrm{~nm} \\
\text { Growth rate }(\AA / \mathrm{s})\end{array}$ \\
\hline 10 & 137 & 0.7 & 369 & 2.0 \\
25 & 155 & 0.8 & 326 & 1.8 \\
50 & 171 & 0.9 & 247 & 1.4 \\
100 & 108 & 0.6 & 306 & 3.7 \\
150 & 181 & 1.0 & 553 & 3.0 \\
\hline
\end{tabular}

position at $520 \mathrm{~cm}^{-1}$ by an amount $\Delta \omega$, which is related to the crystallite size $(\delta)$ through the following equation: $\delta=2 \pi(2.24 / \Delta \omega)^{1 / 2}$ [17]. This confinement model is valid only for small nc-Si with average sizes around $2-10 \mathrm{~nm}$ which correspond to frequency shifts between 500 and $519 \mathrm{~cm}^{-1}$. In our case, the frequency shift was calculated as the difference between the adjusted crystalline TO peak frequency and the TO peak frequency for microcrystalline silicon $\left(520 \mathrm{~cm}^{-1}\right)$. A common form of derivation of the crystalline fraction $X_{C}$ was proposed by Bustarret et al. [18], $X_{C}=I_{C} /\left(I_{C}+y I_{a}\right)$, where $I_{C}$ is the sum of the areas corresponding to the nanocrystalline and microcrystalline fractions and $y$ is a function of the crystallites size $(L)$ expressed in angstroms $y(L)=0.1+\exp -(L / 250)$. In our case, to calculate the crystalline fraction, we used the Brunel model with $y \cong 0.9$, because the HRTEM results show that the crystallites average sizes are smaller than $10 \mathrm{~nm}$. The Raman spectra of the samples were analyzed by deconvolution into 3 Gaussian peaks corresponding to the microcrystalline $\left(520 \mathrm{~cm}^{-1}\right)$, nanocrystalline (adjusted between $\left.500-518 \mathrm{~cm}^{-1}\right)$, and amorphous phase $\left(480 \mathrm{~cm}^{-1}\right)$, and the areas of each peak were used to calculate $I_{C}$ and $I_{a}$. An example of the deconvolution of Raman spectra is shown in the insert of Figure 2.

Figure 2 shows representative Raman spectra of films deposited with different flow rate ratios $R$ and different RF power. The change in the crystalline fraction of the samples as a function of the growth parameters is clear from an inspection of the spectra. It can be seen from Figures 2(a)and 2(b) that the films grown with $R=0.05$ tend to be more nanocrystalline than the films grown with $R=0.1$. The highest nanocrystallinity is achieved when $R$ is 0.05 and the RF power is 50 watt. Increasing $R$ to 0.1 causes the crystalline peak to diminish considerably, while the amorphous peak becomes prominent. Figure 3(a) shows the volumetric crystalline fractions calculated from the Raman spectra deconvolution for the different samples as a function of the RF power. The crystalline fraction for the samples deposited with $R=0.1$ does not present a significant variation with RF power. On the other hand, $X_{C}$ increases, reaches a maximum, and then decreases for the samples deposited with $R=0.05$.

From Figure 3(a), we can observe that the hydrogen dilution plays an important role in the crystalline fraction of the pm-Si films. The samples deposited with $R=0.05$ have a higher hydrogen dilution and the changes in $X_{C}$ are more pronounced with RF power. It is well established that a higher $\mathrm{H}_{\mathrm{at}}$ dilution enhances crystallization in $\mathrm{SiH}_{4} / \mathrm{H}_{2}$ systems as has been reported previously for other deposition conditions of polymorphous and microcrystalline silicon ( $\mu \mathrm{c}-\mathrm{Si}$ ) thin films by PECVD $[16,19]$. However, in the case of chlorine-related silicon precursors, there is a competition between this process and higher chlorine incorporation in the growing surface, which depends on the relative amount of chlorine related species and atomic hydrogen. SiCld and $\mathrm{SiHCl}$ complexes incorporated in several monolayers near the growing surface include high amounts of bond angle distortion and bond length fluctuation, which can induce a higher degree of disorder and stress in the amorphous silicon network [20]. This can explain why $X_{C}$ decreases with increasing RF power for $R=0.05$ and why $X_{C}$ is smaller in the samples prepared with a higher amount of chlorinerelated precursors $(R=0.1)$.

Figures 3(b) and 3(c) show a comparison of the average crystallites sizes calculated from the shift $\Delta \omega$ of the Raman c-Si peak and the average crystallite size measured from HRTEM images. The values of $\Delta \omega$ are reported in Table 2 for all the samples. It is very important to note the great similitude between the HRTEM and Raman results. For high powers, there is a small difference in the average crystallite size obtained from both techniques. This can be attributed to the broad size distribution that was observed in the samples grown at high RF powers. Since Raman is a macroscopic analysis and HRTEM is nanoscopic, such differences in the average sizes are expected.

Additionally, the Raman spectra allow us to estimate a parameter that provides information about the distortion grade of the amorphous silicon network. This parameter is the Root Mean Square (RMS) bond angle deviation $\left(\Delta \theta_{b}\right)$ of the amorphous matrix (crystalline silicon in the diamond cubic structure has $\left.\Delta \theta_{b}=0^{\circ}\right)$. Beeman et al. [21] proposed a model that relates the Full Width at Half Maximum (FWHM) of the amorphous silicon Raman peak with the RMS bond angle deviation: FWHM $=15+6 \Delta \theta_{b}$. Based on different models of relaxed amorphous silicon networks, this parameter is in the range $7.7^{\circ} \leq \Delta \theta_{b} \leq 10.5^{\circ}$. This relaxed amorphous silicon networks can be obtained only after annealing of the deposited amorphous silicon films at temperatures between $170^{\circ} \mathrm{C}-200^{\circ} \mathrm{C}$. On the other hand, according to Beeman et al. the range between $0^{\circ}<\Delta \theta_{b} \leq$ $6.6^{\circ}$ cannot be modeled by a homogeneous network so it is related to the amorphous/crystalline transition [21]. 
TABLE 2: Results of the analysis of Raman spectra: crystalline fraction, bond angle deviation, nanocrystalline Si peak shift, and calculated crystallites average size for samples deposited with different $R=\operatorname{Fr}\left[\mathrm{SiH}_{2} \mathrm{Cl}_{2}\right] / \mathrm{Fr}\left[\mathrm{H}_{2}\right]$ and $\mathrm{RF}$ powers. The crystallites average size measured by HRTEM is included for comparison.

\begin{tabular}{|c|c|c|c|c|c|c|}
\hline & RF power $(\mathrm{W})$ & $\begin{array}{l}\text { Crystalline } \\
\text { fraction }(\%)\end{array}$ & $\begin{array}{c}\text { Bond angle } \\
\text { deviation } \Delta \theta_{b}\left({ }^{\circ}\right)\end{array}$ & $\begin{array}{c}\text { c-Si shift } \\
\Delta \omega\left(\mathrm{cm}^{-1}\right)\end{array}$ & $\begin{array}{l}\text { Calculated size } \\
\text { by model }(\mathrm{nm})\end{array}$ & HRTEM size $(\mathrm{nm})$ \\
\hline \multirow{5}{*}{$R=0.1$} & 10 & 18.1 & 10.0 & 12.3 & $2.7 \pm 0.5$ & $2.4 \pm 0.4$ \\
\hline & 25 & 19.0 & 8.5 & 14.2 & $2.5 \pm 0.5$ & $2.6 \pm 0.5$ \\
\hline & 50 & 27.4 & 8.1 & 9.0 & $3.1 \pm 0.5$ & $2.8 \pm 0.8$ \\
\hline & 100 & 22.1 & 8.6 & 14.0 & $2.5 \pm 0.5$ & $2.8 \pm 1.0$ \\
\hline & 150 & 21.9 & 8.5 & 7.4 & $3.4 \pm 0.5$ & $4.2 \pm 1.6$ \\
\hline \multirow{5}{*}{$R=0.05$} & 10 & 13.1 & 7.8 & 14.8 & $2.4 \pm 0.5$ & $2.5 \pm 1.2$ \\
\hline & 25 & 26.6 & 8.7 & 8.6 & $3.2 \pm 0.5$ & $3.7 \pm 1.6$ \\
\hline & 50 & 53.9 & 7.3 & 8.8 & $3.2 \pm 0.5$ & $3.9 \pm 1.7$ \\
\hline & 100 & 12.3 & 7.6 & 5.0 & $4.2 \pm 0.5$ & $4.3 \pm 2.0$ \\
\hline & 150 & 25.0 & 8.2 & 4.4 & $4.5 \pm 0.5$ & $2.9 \pm 1.3$ \\
\hline
\end{tabular}

The FWHM values of the deconvoluted Raman peak at $480 \mathrm{~cm}^{-1}$ are between 59 and $75 \mathrm{~cm}^{-1}$ for our polymorphous silicon thin films, which give values of $\Delta \theta_{b}$ between 7.3 and 10 degrees (see values in Table 2). This indicates that our films have a bond distortion level comparable to relaxed amorphous silicon networks or in some samples (deposited with $R=0.05$ ) minor to that obtained in standard silicon amorphous thin films [21]. We believe that this low level of disorder in the amorphous matrix is caused by the existence of crystalline zones, which tend to create a short-range order around nanocrystals boundaries. Besides, the local heating associated to the chlorine chemistry of the deposition process can contribute to stress release and local relaxation of the growing film. Having a relaxed amorphous silicon matrix is important because it can give a greater stability to $\mathrm{pm}-\mathrm{Si}$ thin films under the action of external agents (solar radiation and sudden temperature changes), which is a critical issue for applications in solar cells.

3.3. UV-Vis Absorption. In order to link the crystalline fraction or the internal structure of polymorphous silicon thin films with their optical properties, transmittance measurements of the films grown on the quartz substrates were performed. The effective absorption coefficient $\left(\alpha_{\text {eff }}\right)$ (not absolute) of the films was derived from a simple model starting from the transmittance measurements $(\% T)$ : $\alpha_{\text {eff }}=(1 / d) \ln (100 / \% T)$, where $d$ is the sample thickness in $\mathrm{cm}$. The purpose of calculating this effective absorption coefficient is to compare between the different samples in this work and mainly in the regions of high absorption where the interference phenomena are not as pronounced.

Figures 4(a) and 4(b) show the effective absorption coefficient of the different samples plotted as a function of the photon energy $(h \nu)$ grown with different $R$ and RF powers. This allows us to compare the different absorption properties between the pm-Si samples in the spectral regions that are of interest in photovoltaic materials. One can observe from the figure that the samples deposited at $R=0.05$ present a higher absorption in the UV-visible region (photon energies from 2.0 to $3.5 \mathrm{eV}$ ) than the samples deposited with $R=0.1$. Also, the absorption coefficient changes significantly with the variation of RF power for a given $R$, but this change is not monotonic. On the other hand, the optical gap $E_{g}$ was calculated using the Tauc model [18], which can be applied because in our samples more than $50 \%-80 \%$ corresponds to the amorphous matrix. In this method, $(\alpha h v)^{1 / 2}$ is plotted versus $h v$ and a linear regression is performed in the region around the absorption edge where $(\alpha h \nu)^{1 / 2}$ is proportional to $h \nu$. The energy-axis intercept of this line is associated with the optical band gap $E_{g}$. With this approximation, an effective optical gap is obtained that is representative of the whole material. The optical band gap $E_{g}$ was obtained in the region of high absorption where the interference phenomenon due to thickness is not significant. $E_{g}$ is plotted versus RF power in the inserts of Figure 4, along with the crystalline fraction in order to relate them. The Tauc band gap of our pm-Si films varies between 1.78 and $2.37 \mathrm{eV}$. This band gap is higher than that of conventional a-Si:H $(1.5-1.8 \mathrm{eV})$.

The different absorption properties of the samples can be explained by a combination of the heterogeneous microstructure of the films (amorphous matrix and nanocrystallites) characterized by $X_{C}$ and quantum confinement effects in the nanocrystallites which depend on the average size and size distribution of the samples. It is important to note that neither the optical band gap $\left(E_{g}\right)$ nor the nanocrystalline fraction percent $\left(X_{C}\right)$ varies monotonically with the variation of $R$ and RF power. This is explained again in terms of the chlorine chemistry introduced in the deposition process. The competition between $\mathrm{H}_{\text {at }}$ generation and chlorine related species can give rise to a growth regime and/or an etching regime that generates different microstructures as has been explained in this work and in previous works $[10,13,14,20]$. For the sample grown with higher hydrogen dilution, $E_{g}$ and $X_{C}$ have a similar behavior as a function of the RF power. However, this trend is not so explicit for the samples grown with $R=0.1$ that have, in comparison, a smaller nanocrystalline fraction. On the other hand, both for $R=0.05$ and $R=0.1$, the optical 
band gap decreases at high RF powers. This red-shift can be associated to quantum size effects, since the average size and size distribution of the samples get larger with increasing RF power.

The differences in the absorption properties of these films can be advantageous for silicon solar cells applications. For a given photon energy, the effective absorption coefficient can be as different as one order of magnitude for films grown with different conditions. For example, at $2.3 \mathrm{eV}$ or $540 \mathrm{~nm}$ corresponding to the peak of the solar spectrum, the sample grown with $R=0.1$ and $50 \mathrm{~W}$ has $\alpha_{\text {eff }}=$ $0.97 \times 10^{4} \mathrm{~cm}^{-1}$ while the sample grown with $R=0.05$ and $100 \mathrm{~W}$ has $\alpha_{\text {eff }}=1.05 \times 10^{5} \mathrm{~cm}^{-1}$. Such a possibility of changing the absorption could be exploited in a PIN type solar cell structure where one could use different pm-Si thin films, one as emitter and the other as intrinsic (absorbent) materials, respectively. This has the additional advantages of material compatibility during device processing and increased stability of polymorphous silicon with respect to conventional amorphous silicon.

\section{Conclusions}

Different crystalline fractions and average sizes of silicon nanocrystals embedded in amorphous silicon thin films matrix can be obtained by changing the RF power and the dichlorosilane to hydrogen flow rate ratio during the PECVD process. The HRTEM images showed that the biggest nanocrystalline inclusions have irregular shapes supporting the hypothesis that they are, in fact, crystallized areas and not crystallites generated in the plasma region and then embedded in the amorphous matrix. Also, the crystallites size distribution broadens with increasing RF power. The average size obtained from the analysis of Raman spectra are in good agreement with the HRTEM measurements, evidencing that this information can be obtained in a simple way by Raman spectroscopy which is a trustworthy, macroscopic and nondestructive technique. Raman measurements also indicate that the films grown starting from dichlorosilane have a lower bond distortion level comparable to relaxed amorphous silicon networks with annealing, which is important for the stability of the films. The absorption properties and the optical band gap depend on the different internal structures obtained as a function of the growth parameters. For the specific growth conditions corresponding to $R=0.05$ (higher hydrogen dilutions), the crystalline fraction and the optical band gap have the same variation trend as a function of the RF power.

\section{Acknowledgments}

The authors acknowledge partial financial support for this work from the DGAPA-UNAM PAPIIT Projects no. IN116409-2 and IN115909-2, the CONACyT México under project no. 48970, and the ICyTDF project no. PIFUTP08143. A. Remolina thanks the scholarship grant by CLAFBrasil and ICyTDF-México DF. M. F. García-Sánchez acknowledges the postdoctoral fellowship from ICyTDFMéxico DF. The authors are grateful to Dr. J.C. Alonso and Dr. A. Ortiz for the use of laboratory facilities for sample preparation, M. T. Vázquez Mejía, O. Jimenez, J. Camacho, and C. Gonzalez for technical and information services.

\section{References}

[1] M. A. Green, Third Generation Photovoltaics, vol. 12 of Springer Series in Photonics, Springer, Amsterdam, The Netherlands, 2003.

[2] J. Poortmans and V. Arkhipov, Thin Film Solar Cells Fabrication, Characterization and Applications, John Wiley \& Sons, Sussex, UK, 2006.

[3] D. L. Staebler and C. R. Wronski, "Optically induced conductivity changes in discharge-produced hydrogenated amorphous silicon," Journal of Applied Physics, vol. 51, no. 6, pp. 3262-3268, 1980.

[4] P. Roca i Cabarrocas, A. Fontcuberta I Morral, and Y. Poissant, "Growth and optoelectronic properties of polymorphous silicon thin films," Thin Solid Films, vol. 403-404, pp. 39-46, 2002.

[5] T. Nguyen-Tran, V. Suendo, P. Roca I Cabarrocas, L. N. Nittala, S. N. Bogle, and J. R. Abelson, "Fluctuation microscopy evidence for enhanced nanoscale structural order in polymorphous silicon thin films," Journal of Applied Physics, vol. 100, no. 9, Article ID 094319, 2006.

[6] K. Morigaki, K. Takeda, H. Hikita, and P. Roca I Cabarrocas, "Light-induced defect creation in hydrogenated polymorphous silicon," Materials Science and Engineering B, vol. 121, no. 1-2, pp. 34-41, 2005.

[7] A. Gajović, D. Gracin, K. Juraić, J. Sancho-Parramon, and M. Čeh, "Correlating Raman-spectroscopy and highresolution transmission-electron-microscopy studies of amorphous/nanocrystalline multilayered silicon thin films," Thin Solid Films, vol. 517, no. 18, pp. 5453-5458, 2009.

[8] D. Han, K. Wang, J. M. Owens et al., "Hydrogen structures and the optoelectronic properties in transition films from amorphous to microcrystalline silicon prepared by hot-wire chemical vapor deposition," Journal of Applied Physics, vol. 93, no. 7, pp. 3776-3783, 2003.

[9] M. Ledinský, A. Vetushka, J. Stuchlík et al., "Crystallinity of the mixed phase silicon thin films by Raman spectroscopy," Journal of Non-Crystalline Solids, vol. 354, no. 19-25, pp. 22532257, 2008.

[10] A. Remolina, B. M. Monroy, M. F. García-Sánchez et al., "Polymorphous silicon thin films obtained by plasma-enhanced chemical vapor deposition using dichlorosilane as silicon precursor," Nanotechnology, vol. 20, no. 24, Article ID 245604, 2009.

[11] B. M. Monroy, G. Santana, J. Fandiño, A. Ortiz, and J. C. Alonso, "Growth of silicon nanoclusters on different substrates by plasma enhanced chemical vapor deposition," Journal of Nanoscience and Nanotechnology, vol. 6, no. 12, pp. 3752-3755, 2006.

[12] B. M. Monroy, G. Santana, A. Benami et al., "Photoluminescence of as-grown silicon nanocrystals embedded in silicon nitride: influence of atomic hydrogen abundance," Journal of Nanoscience and Nanotechnology, vol. 9, no. 5, pp. 2902-2909, 2009.

[13] J. C. Alonso, A. Ortiz, and C. Falcony, "Low temperature SiO films deposited by plasma enhanced techniques," Vacuum, vol. 43, no. 8, pp. 843-847, 1992. 
[14] G. Viera, S. Huet, M. Mikikian, and L. Boufendi, "Electron diffraction and high-resolution transmission microscopy studies of nanostructured Si thin films deposited by radiofrequency dusty plasmas," Thin Solid Films, vol. 403-404, pp. 467-470, 2002.

[15] H. Shirai, "Role of chlorine in the nanocrystalline silicon film formation by rf plasma-enhanced chemical vapor deposition of chlorinated materials," Thin Solid Films, vol. 457, no. 1, pp. 90-96, 2004.

[16] E. A. G. Hamers, A. Fontcuberta i Morral, C. Niikura, R. Brenot, and P. Roca i Cabarrocas, "Contribution of ions to the growth of amorphous, polymorphous, and microcrystalline silicon thin films," Journal of Applied Physics, vol. 88, no. 6, pp. 3674-3688, 2000.

[17] H. Richter, Z. P. Wang, and L. Ley, "The one phonon Raman spectrum in microcrystalline silicon," Solid State Communications, vol. 39, no. 5, pp. 625-629, 1981.

[18] E. Bustarret, M. A. Hachicha, and M. Brunel, "Experimental determination of the nanocrystalline volume fraction in silicon thin films from Raman spectroscopy," Applied Physics Letters, vol. 52, no. 20, pp. 1675-1677, 1988.

[19] A. Matsuda, "Growth mechanism of microcrystalline silicon obtained from reactive plasmas," Thin Solid Films, vol. 337, no. 1-2, pp. 1-6, 1999.

[20] G. Santana, J. Fandiño, A. Ortiz, and J. C. Alonso, "Low temperature-low hydrogen content silicon nitrides thin films deposited by PECVD using dichlorosilane and ammonia mixtures," Journal of Non-Crystalline Solids, vol. 351, no. 1011, pp. 922-928, 2005.

[21] D. Beeman, R. Tsu, and M. F. Thorpe, "Structural information from the Raman spectrum of amorphous silicon," Physical Review B, vol. 32, no. 2, pp. 874-878, 1985. 

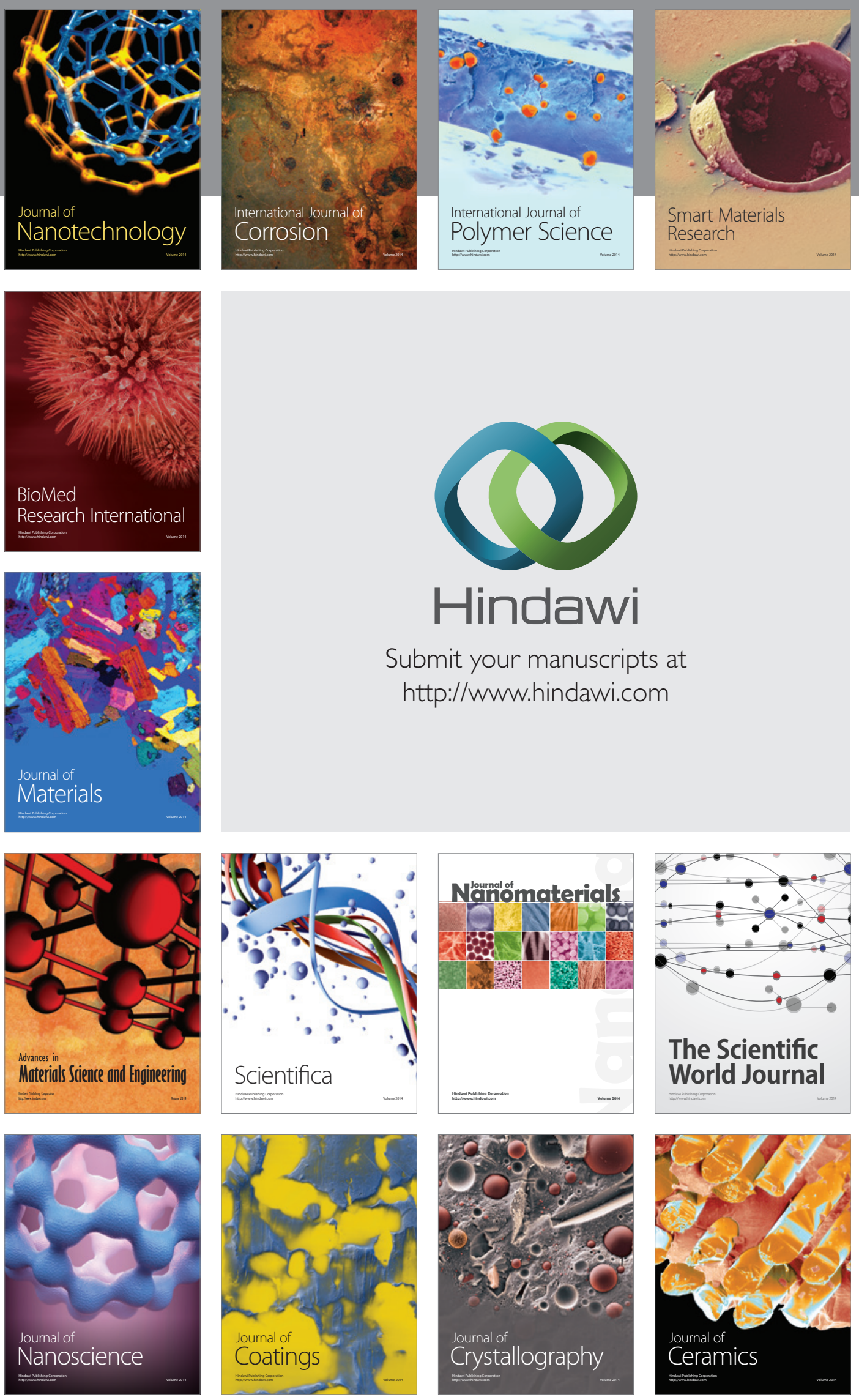

The Scientific World Journal

Submit your manuscripts at

http://www.hindawi.com

\section{World Journal}

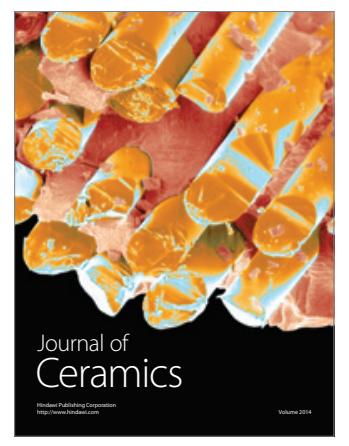

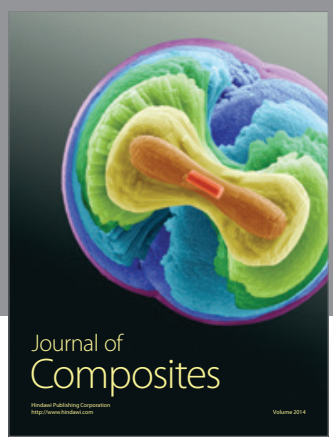
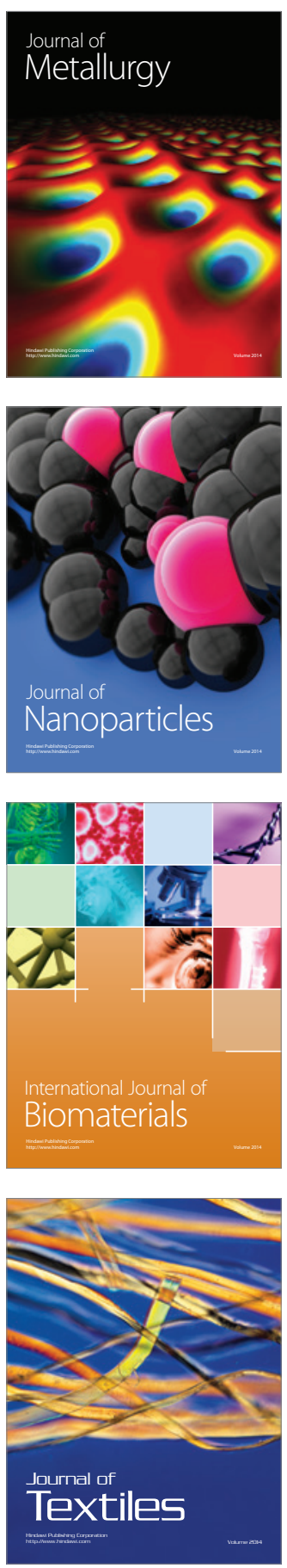